Patient 2 abuses alcohol when hypomanic as a symptom of his illness. He does not abuse it when depressed or euthymic.

Patient 3 has the differential diagnosis of drug induced psychosis at every admission and probably fits into the category described by Professor Cohen. Neuroleptic medication is usually prescribed symptomatically on admission and is effective in reducing psychotic features and behavioural disturbances. I know of no general psychiatric facility which could cope with acutely psychotic young men without some form of chemical sedation. It would be a misuse of the forensic service even if they could handle the numbers involved.

Most drug abusers tend to "mature out" from drug abuse over a period of up to ten years. This provides the rationale for maintenance treatment of addicts, keeping them as healthy as possible, out of trouble with police, and in contact with a trusted psychiatric service for when they are willing to accept help. Surely the drug abuser who becomes psychotic is most in need of this continuing support?

Wirral Hospital

Mavis Evans

Bebington, Wirral

Merseyside L63 4JY

\section{Research accreditation of seniors?}

\section{DeAR SirS}

We read with interest the report by Bartlett and Drummond (Psychiatric Bulletin June 1992, 16, 361-362) concerning the difficulties one of their registrars had with a research project of theirs. One of us (Kerwin, 1992) recently made a plea for proper research training of consultants before they are allowed to supervise juniors. This was a somewhat tongue in cheek letter (tit for tat for the " $T$ " psych accreditation for clinical academics) but clearly this case highlights the need for ensuring that consultants should also be properly trained to supervise research.

Registrar research need not be difficult so long as consultants ensure success by advising on parsimonious and achievable studies. Drs Bartlett and Drummond asked their hapless registrar to perform a "... randomised double blind, double dummy, parallel group comparison of trazodone and clomipramine as an adjunct to behaviour therapy in the treatment of non depressed subjects with primary obsessive compulsive disorder!"

Maybe we really should insist on research accreditation of seniors?

R. KERWIN

Institute of Psychiatry

De Crespigny Park

London SES 8AF

\section{Reference}

KERWIN, R. (1992) Raw deal for academics, British Medical Journal 304, 1058

\section{Reply}

DeAR SirS

We would like to thank Drs Kerwin and Pilowsky for their interest in our article. Regrettably, they appear to have misunderstood our purpose and, equally regrettably, to have resorted to an implicit attack on our research credentials. We will not address the second of these two criticisms. However, we would like to emphasise that in practice research can be hampered by problems, both within and outside the researcher's control. Registrars, at an early stage of a research career, are particularly vulnerable to such difficulties, even when appropriately supervised by experienced senior academics. Furthermore, the writing up of research within the style favoured by the 'medical model' encourages authors to be less than frank about the practical aspects of research and to disguise deficiencies in their 'end product'. We have 'come out' about the reality behind much of this type of research.

A. E. A. BARTLETT
L. M. DRUMMOND
St George's Hospital Medical School
Cranmer Terrace
London SW170RE

\section{Overseas doctors - training ethos}

I have been closely following the correspondence pertaining to overseas doctors and their training requirements. Each author (Matthew, O'Dwyer, Zaffar, Gandhi) and the Royal College of Psychiatrists (Psychiatric Bulletin, 1991, 15, 699-700; 1992, 16, 231-232; 1992, 16, 446-447) has made pertinent points.

The arguments are self-fulfilling towards a distinct symbiotic relationship between overseas doctors and the NHS of the UK. The majority of overseas doctors are keen to work in the UK for a British degree, and in return the NHS fulfils its manpower requirements, thus serving the philosophy of Achieving a Balance. The NHS is a beneficiary of highly motivated and well-qualified manpower obtained through the Overseas Doctors Training Scheme. 'Overseas doctors' are in the prime of their youth, trained at the expense of the developing world, contributing their share to the national exchequer by paying taxes and National Insurance contributions. An 'overseas doctor' is offered a training post which helps fulfil the statutory requirements needed to obtain a higher British qualification in psychiatry, which is a powerful tool to face stiff 
professional competition and bureaucracy on their return home. I agree that 'overseas doctors' are not a homogeneous group and generalisations cannot be drawn, but one cannot deny that higher qualifications are a must for professional survival anywhere.

What should be the common by-product of this symbiotic relationship which is ignored by all concerned parties? The answer lies in the betterment of mental health and its services in the developing world. Overseas trainees (irrespective of country) and the Royal College of Psychiatrists should both aim to achieve this common objective.

Overseas doctors should during their stay in the UK develop their initiative and creative instincts, and improve their theoretical education (which has too great a dependence on the retention of facts), by learning to assemble knowledge and use it. The College should strive to improve and standardise the quality of training imparted to overseas doctors (Mbwambo et al, 1992). The developing countries represent a unique social and human laboratory and a vast area of the 'universe' for renewed scientific consideration (Lambo, 1982).

'Achieving a balance' with a certain standard of quality and quantity of overseas trainees, and attaining the MRCPsych examinations for future survival, are but momentary phases of glory, both for the College and the trainees. Let us as professionals be honest in our long-term intentions and mutually assist in this novel approach to training by helping to make the ODTS a means to a nobler end: the alleviation of mental health suffering in the developing world.

Royal Shrewsbury Hospital-Shelton

DARRYL J. BRITTO Shrewsbury SY3 8DN

\section{References}

Lambo, T. (1982) Current status of psychiatry. In Psychiatrists on Psychiatry (ed. M Shepherd). Cambridge University Press.

Mbwambo, J., Appleby, L. \& Gater, R. (1992) The training of psychiatrists for the developing world. Psychiatric Bulletin, 16, 352-354.

\section{DeAr Sirs}

Having recently spent time in an African country considering, among other things, postgraduate training in psychiatry, I read with interest the recent article on training psychiatrists for the developing world (Psychiatric Bulletin, June 1992, 16, 352-354) and Dr Gandhi's letter on overseas doctors (Psychiatric Bulletin, July 1992, 16, 446-447).

The ODTS offers a way for doctors from poorer countries to gain experience unavailable in their own countries and it also provides much needed manpower at a junior level for our own NHS. Both the article and the letter make the point that the training needs of the doctors on the scheme vary and that there should be greater flexibility in meeting individual requirements. There are, however, at least four elements each with its own agenda, namely the College, the NHS, the country of origin, and the trainees, so it is hardly surprising that criticism of the scheme should emerge and ways to balance and reorganise it should be sought.

If the scheme is truly to address the training needs of doctors from overseas then it would be advantageous for the trainers to have knowledge of services overseas, and while this is not immediately possible, some exchange of trainees might be explored. From my own experience in Zimbabwe I am convinced that valuable training opportunities exist, particularly at SR level, and the ODTS might develop a two way movement of trainees, forging links with particular centres in developing countries, perhaps having a list of approved centres that would be recognised for higher training purposes by the College.

There must be a role for a discipline such as psychiatry to foster understanding between cultures through an exchange of ideas and people, trying to overcome the boundaries that nations build between themselves.

\section{Paddington Community Hospital London W9 2BB}

J. Piachaud

\section{DeAr Sirs}

I wish to comment on the article by Mbwambo and colleagues about the training of psychiatrists for the developing world (Psychiatric Bulletin, June 1992, 16, 352-354) which opens a number of valid questions about the training of overseas doctors. I appreciate that countries from the developing world are very different in their needs and existing services. Egypt, with over 300 psychiatrists and a substantial number of religious and faith healers, would be very different from countries with a handful of psychiatrists and lacking an organised medical service.

The needs of individual trainees also differ. His long-term position in his home country may be in a teaching, research or clinical service. There is no possible way of improving the current training of doctors in a manner that would fulfil all the needs of different trainees from the developing world.

Rather than attempting to change the current training schemes in the UK, one should stress more the importance of communication between the UK training centre and local psychiatrists and health planners in the developing countries, to discuss the specific needs and expectations of individual trainees. The months preceding the trainee's arrival in the UK are usually spent sorting out GMC papers, 